Review Article

\title{
Diplazium esculentum (Retz.) Sw.: Ethnomedicinal, Phytochemical, and Pharmacological Overview of the Himalayan Ferns
}

\author{
Prabhakar Semwal (D), ${ }^{1,2}$ Sakshi Painuli, ${ }^{1,3}$ Kartik M. Painuli, ${ }^{4}$ Gizem Antika, ${ }^{5}$ \\ Tugba Boyunegmez Tumer, ${ }^{6}$ Ashish Thapliyal, ${ }^{1}$ William N. Setzer, ${ }^{7,8}$ Miquel Martorell ${ }^{\circ},{ }^{9}$ \\ Mohammed M. Alshehri $\mathbb{D}^{10}{ }^{10}$ Yasaman Taheri, ${ }^{11}$ Sevgi Durna Daştan, ${ }^{12,13}$ \\ Seyed Abdulmajid Ayatollahi $\mathbb{D}^{1},{ }^{11,14,15}$ Anka Trajkovska Petkoska, ${ }^{16}$ Javad Sharifi-Rad (D), 11 \\ and William C. Cho ${ }^{17}$
}

${ }^{1}$ Department of Biotechnology, Graphic Era University, Dehradun, Uttarakhand, India

${ }^{2}$ Uttarakhand State Council for Science and Technology, Dehradun, Uttarakhand, India

${ }^{3}$ Himalayan Environmental Studies and Conservation Organization, Dehradun, Uttarakhand, India

${ }^{4}$ Uttarakhand Ayurved University, Gurukul Campus (Haridwar), Uttarakhand, India

${ }^{5}$ Graduate Program of Molecular Biology and Genetics, Institute of Natural and Applied Sciences,

CanakkaleOnsekiz Mart University, Canakkale, Turkey

${ }^{6}$ Department of Molecular Biology and Genetics, Faculty of Arts and Science, CanakkaleOnsekiz Mart University, Canakkale, Turkey

${ }^{7}$ Department of Chemistry, University of Alabama in Huntsville, Huntsville, AL 35899, USA

${ }^{8}$ Aromatic Plant Research Center, 230 N 1200 E, Suite 100, Lehi, UT 84043, USA

${ }^{9}$ Department of Nutrition and Dietetics, Faculty of Pharmacy, And Centre for Healthy Living, University of Concepción, 4070386 Concepción, Chile

${ }^{10}$ Pharmaceutical Care Department, Ministry of National Guard-Health Affairs, Riyadh, Saudi Arabia

${ }^{11}$ Phytochemistry Research Center, Shahid Beheshti University of Medical Sciences, Tehran, Iran

${ }^{12}$ Department of Biology, Faculty of Science, Sivas Cumhuriyet University, 58140 Sivas, Turkey

${ }^{13}$ Beekeeping Development Application and Research Center, Sivas Cumhuriyet University, 58140 Sivas, Turkey

${ }^{14}$ Department of Pharmacognosy and Biotechnology, School of Pharmacy, Shahid Beheshti University of Medical Sciences, Tehran, Iran

${ }^{15}$ H.E.J. Research Institute of Chemistry, International Center for Chemical and Biological Sciences, University of Karachi, Karachi 75270, Pakistan

${ }^{16}$ Faculty of Technology and Technical Sciences, St. Kliment Ohridski University-Bitola, Dimitar Vlahov, 1400 Veles, North Macedonia

${ }^{17}$ Department of Clinical Oncology, Queen Elizabeth Hospital, Kowloon, Hong Kong

Correspondence should be addressed to Javad Sharifi-Rad; javad.sharifirad@gmail.com and William C. Cho; chocs@ha.org.hk

Received 1 June 2021; Revised 7 August 2021; Accepted 13 August 2021; Published 3 September 2021

Academic Editor: Anderson J. Teodoro

Copyright (c) 2021 Prabhakar Semwal et al. This is an open access article distributed under the Creative Commons Attribution License, which permits unrestricted use, distribution, and reproduction in any medium, provided the original work is properly cited.

The genus Diplazium (family: Athyriaceae) comprises approximately 350 species of pteridophytes. Diplazium esculentum (Retz.) Sw. is an important member of this genus and commonly known as a wild vegetable in the Himalayan and sub-Himalayan communities. According to the literature analysis, D. esculentum was traditionally used for the prevention or treatment of several diseases such as diabetes, smallpox, asthma, diarrhea, rheumatism, dysentery, headache, fever, wounds, pain, measles, 
hypertension, constipation, oligospermia, bone fracture, and glandular swellings. Various extracts of D. esculentum were evaluated to elucidate their phytochemical and pharmacological activities. A wide array of pharmacological properties such as antioxidant, antimicrobial, antidiabetic, immunomodulatory, CNS stimulant, and antianaphylactic activities have been recognized in different parts of $D$. esculentum. The review covers a systematic examination of pharmacognosy, phytochemistry, and pharmacological applications of D. esculentum, but scientifically, it is not fully assessed regarding complete therapeutic effects, toxicity, and safety in the human body. The published literature on D. esculentum and its therapeutic properties were collected from different search engines including Wiley online, PubMed, Springer Link, Scopus, Science Direct, Web of Science, Google Scholar, and ACS publications by using specific terms such as "Diplazium esculentum, bioactive compounds, biological activities and health benefits" from 1984 to 2021 (March). Therefore, further studies are required to identify the detailed action mechanism of D. esculentum in vitro/in vivo, and also, more studies should focus on conservation, cultivation, and sustainable utilization of the species.

\section{Introduction}

The Himalayan botanicals are well known to produce wide variety of secondary metabolites due to critical climatic conditions [1-4]. These botanicals, including wild plants, have a significant role in food security and socio-economic development of the region $[5,6]$. Moreover, these botanicals are locally utilized for food resources, medicines, and other purposes due to the presence of numerous bioactive compounds and high nutritional value $[7,8]$. With recent developments in science and technology, the importance of wild plants has been identified as a possible source of nutraceuticals and/or functional foods [9].

Among several high valued functional foods, Diplazium esculentum is one of the important species of wild ferns, which is frequently consumed by people living in the hilly areas; it is not growing on much higher altitude. D. esculentum ( $n=41$ chromosomes, grade of polyploidy = diploid) is utilized as a traditional vegetable in the Himalayan communities [10]. It is an important member of the genus Diplazium which comprises around $~ 350$ species of pteridophytes, mainly distributed in Asia and Oceania [11].

Specifically, D. esculentum is distributed through different parts of the globe including Cambodia, China, India, Indonesia, Japan, Malaysia, Papua New Guinea, Pakistan, Philippines, Singapore, Taiwan, Thailand, Vietnam, and Bangladesh. It grows on the banks of rivers and streams, canals, marshy areas, and hills with an altitudinal range up to 2,300 meters $[12,13]$.

It is locally known by different names such as English: vegetable fern; India: dung-kek, kari-welli-panna-maravara, kasrot, kukari-sag, mairungshai, para-panna-maravara, linguda, kathura; Japan: Kuware-shida; Malay: paku, pakutanjong; Nepali: paninyuro, piraunli; Papua New Guines: sigogo; Philippines: Pako; Thai: kut-kin; and Bangladesh: Dheki Shak [14].

Traditionally, D. esculentum is being used in the treatment of various ailments (as shown in Figure 1) such as diabetes, smallpox, asthma, diarrhea, rheumatism, dysentery, headache, fever, wounds, pain, measles, high blood pressure, constipation, oligospermia, bone fracture, glandular swellings, and skin-related diseases by the different communities in India and other countries [15-21].

Recently, a few researchers have validated its nutraceutical and pharmacological properties by using in vitro and in vivo models/methods. For the development of evidencebased medicine, a critical investigation of current knowledge is required regarding ethnopharmacology, chemical composition, biological activities, and possible side effects of the species. Additionally, D. esculentum belongs to the least concern category under International Union for Conservation of Nature (IUCN) 2021-1 (https://www.iucnredlist.org/ species/194150/8883499) and needs more attention. Therefore, in this manuscript, we reviewed and discuss the recent scientific information conducted so far on D. esculentum, which includes its pharmacognosy, phytochemistry, and pharmacology.

\section{Pharmacognosy}

2.1. Traditional Uses. Traditionally, D. esculentum is one of the most popular vegetables consumed in different parts of the globe, namely, India, Philippines, Nepal, China, Thailand, Indonesia, etc. The literature has revealed that D. esculentum is still being used by different communities for the treatment of several diseases including diabetes, smallpox, asthma, diarrhea, rheumatism, dysentery, headache, fever, wounds, pain, measles, and high blood pressure. The detailed information on the traditional uses of the species is summarized in Table 1. Additionally, this species is collected in large amounts and marketed by the rural and tribal communities of India for their livelihood enhancement [22]. The Mishing community of Assam (State of the Indian republic) used it essentially in the religious ceremony of the dead person [23].

2.2. Proximate and Mineral Composition. The nutritional value of any food substance can be analyzed by its proximate and mineral composition [24]. Literature-based screening of the proximate composition of $D$. esculentum revealed the presence of lipids, proteins, carbohydrates, vitamins, fiber, etc., while mineral composition possesses the presence of essential micro and macro compounds [25-35]. The comparative analyses of proximate and mineral composition of D. esculentum are presented in Tables 2 and 3.

\section{Bioactive Compounds}

Traditionally, botanicals are being widely used to cure various ailments due to the presence of high-valued bioactive compounds [61, 62]. Literature-based screening for bioactive compounds of $D$. esculentum revealed the presence of 


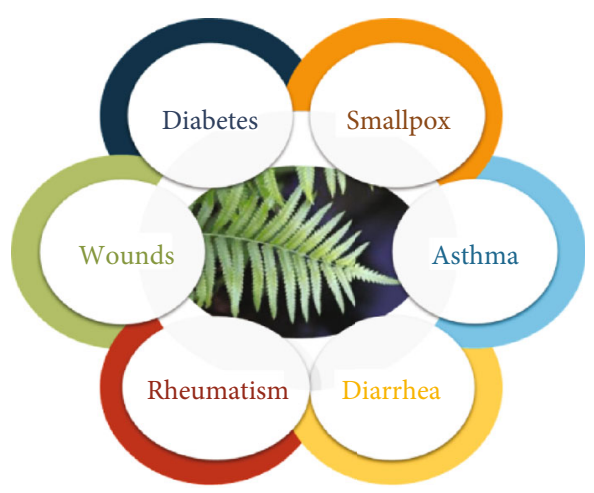

FIgUre 1: Traditional uses of Diplazium esculentum.

alkaloids, flavonoids, glycosides, phenolic, tannins, terpenoids, steroids, carbohydrates, fats, and oils in different solvent systems [2, 35, 46, 50, 60, 63-69].

In the study of Essien and coworkers [16], the chemical composition of essential oil isolated from D. esculentum leaves and the major volatile compounds were identified as $\beta$-pinene (17.2\%), $\alpha$-pinene (10.5\%), caryophyllene oxide (7.5\%), sabinene (6.1\%), and 1,8-cineole (5.8\%) (Figure 2). The essential oil of this species was composed of monoterpene hydrocarbons, oxygenated sesquiterpenoids, sesquiterpene hydrocarbons, oxygenated monoterpenoids, and nonterpene derivatives.

Few compounds such as ascorbic acid [70], eriodictyol 5$O$-methyl ether 7-O- $\beta$-D-xylosyigalactoside [71], tannins and phytates [72], $\alpha$-tocopherol [73], quercetin [74, 75], pterosin [75], ptaquiloside [76], terpene, hopan-triterpene lactone [51], and lutein [77] were also isolated from $D$. esculentum. Additionally, four phenolic compounds ((2R)-3-( $4^{\prime}$ -hydroxyphenyl) lactic acid, trans-cinnamic acid, protocatechuic acid, and rutin) and three ecdysteroids (amarasterone A1, makisterone $\mathrm{C}$, and ponasterone A) were isolated from young fronds of $D$. esculentum collected from Japan [78] while 26 bioactive compounds were identified in the methanolic extracts of young fronds of $D$. esculentum collected from Indian Himalaya [60]. The major compounds present in the species were identified as pentadecanoic acid, $\beta$-sitosterol, neophytadiene, $\alpha$-linolenic acid, methyl palmitate, diisobutyl phthalate, phytol, and 10,12 hexadecadien-1-ol [60]. These all major compounds are shown in Figures 3(a) and 3(b), respectively.

\section{Biological Applications}

Among the functional properties of D. esculentum, the antioxidant, anti-inflammatory, antimicrobial, antidiabetic, and immune-modulatory activities can be considered as potentially contributing to the preventive and pharmacological values of this plant species (Figure 4). The following sections reviewed the abovementioned functional biological activities of different D. esculentum extracts.

4.1. Antioxidant Activities. The botanicals can be considered as safe and cost-effective natural antioxidants capturing free radicals and may help in the prevention and the treatment of different diseases $[79,80]$. Recently, a research group from Indonesia reported that the methanolic extract of $D$. esculentum showed a good antioxidant activity with an $\mathrm{IC}_{50}$ value of $123.95 \mathrm{ppm}$ according to 2,2-diphenyl-1-picrylhydrazyl (DPPH) radical scavenging activity assay [2].

In an in vitro study, the nutritional properties and antioxidant capacity of D. esculentum were evaluated on the ethanol extract of the edible parts. The phytochemical analysis indicated that the ethanolic extract possesses significant concentrations of flavonoids (90.6-144.5 mg QE/gm) and tannins (26.8-57.2 mg GAE/gm). Considerable antioxidant activities of $D$. esculentum were revealed using different antioxidant assays including $\mathrm{DPPH}$ radical scavenging $\left(\mathrm{IC}_{50}=146.51 \mu \mathrm{g} / \mathrm{mL}\right)$, superoxide radical scavenging $\left(\mathrm{IC}_{50}=111.17 \mu \mathrm{g} / \mathrm{mL}\right)$, hydroxyl radical scavenging $\left(\mathrm{IC}_{50}=43.45 \mu \mathrm{g} / \mathrm{mL}\right)$, and reducing power $\left(\mathrm{IC}_{50=} 76.36\right.$ $\mu \mathrm{g} / \mathrm{mL})$ assays.

In another study, the antioxidant activity of D. esculentum, extracted by using pressurized hot water extraction (PHWE) method, was reported [81]. The results demonstrated that the optimum condition for the best antioxidant activity of $\mathrm{PHWE}$ was at $175^{\circ} \mathrm{C}, 21 \mathrm{~min}$ extraction time $(2 \mathrm{~g}$ dried powder in $50 \mathrm{~mL}$ water) in Box-Behnken design. The plant extract showed moderate DPPH scavenging activity $\left(\mathrm{EC}_{50}=1241.14 \mu \mathrm{g} / \mathrm{mL}\right)$. The hydro-alcoholic extract of $D$. esculentum leaf was evaluated for antioxidant activity using the $\mathrm{DPPH}$ and nitric oxide assays [82]. The $\mathrm{IC}_{50}$ value of the plant extract for DPPH and NO inhibition activity was found to be 138.8 and $151.9 \mathrm{mg} / \mathrm{mL}$, respectively.

The methanolic extract of D. esculentum fronds showed promising antioxidant activity using different assays (DPPH, ABTS, NO, metal chelating, and superoxide scavenging activity) [64]. The $\mathrm{IC}_{50}$ values of the plant extract was recorded as 3.8, 4.6, 0.59, and $2.24 \mathrm{mg} / \mathrm{mL}$ for DPPH, ABTS, metal chelating, and superoxide scavenging activity, respectively, while nitric oxide, hydroxyl ion, and FRAP assays were recorded as $100-10000 \mu \mathrm{g} / \mathrm{mL}, 100-10000 \mu \mathrm{g} / \mathrm{mL}$, and $0.095-0.121 \mathrm{mM} \mathrm{Fe}{ }^{2+}$ equivalent. Table 4 includes detailed information about previous antioxidant activities.

4.2. Antimicrobial Activities. Recently, several pathogenic microorganisms have developed antibiotic resistance, and these antibiotics can have undesirable side effects [92]. Thus, researchers are focusing on botanicals for the development of herbal-based antibiotic substitutes [93]. Table 5 includes antimicrobial studies performed with $D$. esculentum. Antimicrobial activity was considered good (minimum inhibitory concentration (MIC) less than $100 \mu \mathrm{g} / \mathrm{mL}$ ), moderate (MIC from 100 to $500 \mu \mathrm{g} / \mathrm{mL}$ ), weak (MIC from 500 to $1000 \mu \mathrm{g} / \mathrm{mL}$ ), or inactive (MIC over $1000 \mu \mathrm{g} / \mathrm{mL}$ ). Inactive results of antimicrobial activities of $D$. esculentum did not included in this study $[46,94]$.

The areal parts of $D$. esculentum were extracted with ethanol to evaluate the antimicrobial properties by using the disk diffusion method. The crude extract showed considerable antimicrobial activity in terms of minimum inhibitory concentration (MIC) and minimum bactericidal concentration $(\mathrm{MBC})$ value. The MIC value was recorded from a range of $200-800 \mu \mathrm{g} / \mathrm{mL}(200 \mu \mathrm{g} / \mathrm{mL}$ (Bacillus cereus), $400 \mu \mathrm{g} / \mathrm{mL}$ 
TABLe 1: Traditional uses of D. esculentum in different regions.

\begin{tabular}{|c|c|c|c|}
\hline Plant part & Ethno-pharmacological uses & Country & References \\
\hline \multirow{7}{*}{$\begin{array}{l}\text { Fronds/leaves/ } \\
\text { areal part/whole } \\
\text { plant }\end{array}$} & Cooked and eaten as a vegetable and in soups to maintain good health. & India, Bangladesh, Thailand & $\begin{array}{c}{[23,36-} \\
38]\end{array}$ \\
\hline & $\begin{array}{l}\text { Tender leaves are cooked with fruit of Dillenia indica and fish and taken } \\
\text { as vegetable. }\end{array}$ & Malaysia, India & {$[39,40]$} \\
\hline & $\begin{array}{l}\text { Hairs are removed, boiled with salt and water until water is evaporated } \\
\text { then fried and eaten as vegetable. }\end{array}$ & India & [41-45] \\
\hline & $\begin{array}{l}\text { Used in headache, pain, fever, wounds, dysentery, glandular swellings, } \\
\text { diarrhea, measles, toothache, high blood pressure, and various skin } \\
\text { infections. Fronds used by pregnant women as protection against } \\
\text { difficult childbirth. Leaf paste is used in the wounded place externally for } \\
\text { the cure of bone fracture. Used as a laxative. Used as insecticides. }\end{array}$ & $\begin{array}{l}\text { Bangladesh, Nigeria, } \\
\text { Indonesia, Nigeria, India, } \\
\text { Philippines }\end{array}$ & $\begin{array}{c}{[15-17} \\
19,46-48]\end{array}$ \\
\hline & $\begin{array}{l}\text { The tender frond is cooked without salt and is consumed with rice for 5- } \\
\qquad 10 \text { days for the treatment of diabetes. }\end{array}$ & India & {$[20]$} \\
\hline & Eaten as highly preferred Koche Sag, Neuro/Niuro vegetable. & Nepal & [49] \\
\hline & Used as vegetable and pickle. & $\begin{array}{l}\text { India, Vietnam, Japan, } \\
\text { Indonesia, Philippines }\end{array}$ & $\begin{array}{l}{[30,31} \\
50-53]\end{array}$ \\
\hline \multirow{3}{*}{ Root } & $\begin{array}{l}\text { About } 20 \mathrm{~g} \text { of fresh root is boiled in } 1 \text { liter of water and reduced to one- } \\
\text { fourth of its volume. } 3 \mathrm{~mL} \text { of this decoction along with } 2 \mathrm{~mL} \text { of honey is } \\
\text { taken orally on an empty stomach twice a day for } 15 \text { days to cure } \\
\text { spermatorrhea. }\end{array}$ & India & [54] \\
\hline & $\begin{array}{l}\text { About } 50 \mathrm{~g} \text { juice obtained from macerated root is fed three times for } \\
\text { human dysentery. Macerated root extract is also useful for the cattle } \\
\text { dysentery. }\end{array}$ & Bangladesh & [55] \\
\hline & $\begin{array}{l}\text { About 2-3 spoonsful of root juice are taken for } 1 / 2 \text { days, or } 1 / 1 / 2 \text { cup of } \\
\text { boiling extract of whole plant is taken thrice daily to treat infections and } \\
\text { used as an antidote. The root paste is used externally for the treatment of } \\
\text { rheumatism and smallpox. Two pills of pulverized root and honey are } \\
\text { taken thrice daily for } 2 \text { weeks for the treatment of oligospermia. }\end{array}$ & Bangladesh & [17] \\
\hline Rhizome & $\begin{array}{l}\text { Decoction of rhizome used as a tonic and also used for the cure of } \\
\text { hemoptysis and cough. }\end{array}$ & India & {$[39,56]$} \\
\hline
\end{tabular}

Table 2: Proximate composition of Diplazium esculentum from different regions.

\begin{tabular}{|c|c|c|c|c|c|c|c|c|c|}
\hline Parameters & $\begin{array}{c}\text { Bangladesh (mg/ } \\
100 \mathrm{~g})[34]\end{array}$ & $\begin{array}{c}\text { Indonesia } \\
(\%)[29]\end{array}$ & $\begin{array}{c}\text { India (\%) } \\
{[26]}\end{array}$ & $\begin{array}{c}\text { India }(\%) \\
{[57]}\end{array}$ & $\begin{array}{c}\text { Philippines } \\
\text { (\%) [33] }\end{array}$ & $\begin{array}{c}\text { Nepal (\%) } \\
{[58]}\end{array}$ & $\begin{array}{c}\text { Indonesia } \\
(\%)[27]\end{array}$ & $\begin{array}{c}\text { India (\%) } \\
{[59]}\end{array}$ & $\begin{array}{c}\text { India }(\%) \\
{[60]}\end{array}$ \\
\hline Moisture (\%) & 8.8 & - & 89.34 & 92.4 & 91.82 & 93.25 & 90.84 & 93.1 & 90.4 \\
\hline Lipid & 2.16 & - & - & - & - & - & - & - & - \\
\hline Protein & 8.73 & $6.20-8.30$ & 3.84 & 31.2 & $0.87-10.67$ & 0.99 & 2.23 & 2.6 & 8.87 \\
\hline Ash & 5.09 & $1.90-2.11$ & 1.33 & 16.2 & $1.42-17.39$ & 1.10 & 1.38 & 1.3 & - \\
\hline $\begin{array}{l}\text { Total } \\
\text { carbohydrate }\end{array}$ & 59.62 & - & - & 44.3 & - & - & - & 1.0 & 18.8 \\
\hline Fiber & 15.59 & - & 5.05 & 4.6 & $0.72-9.06$ & 0.99 & 4.82 & - & 3.1 \\
\hline Fat & - & $0.51-0.68$ & 0.25 & 8.3 & $0.28-3.40$ & 0.15 & 0.04 & 2.0 & 2.5 \\
\hline Water level & - & $2.70-3.08$ & - & - & - & - & - & - & - \\
\hline $\begin{array}{l}\text { Vitamin C } \\
(\mathrm{mg} / 100 \mathrm{~g})\end{array}$ & - & - & 21.38 & 21 & - & 6.20 & - & - & - \\
\hline
\end{tabular}

(Escherichia coli and Aspergillus ochraceus), and $800 \mu \mathrm{g} / \mathrm{mL}$ (Bacillus megaterium)) while $\mathrm{MBC}$ from a range of 800 to $>800 \mu \mathrm{g} / \mathrm{mL} \quad(800 \mu \mathrm{g} / \mathrm{mL}$ (B. cereus, A. ochraceus) and $>800 \mu \mathrm{g} / \mathrm{mL}$ (B. megaterium, E. coli)), respectively [95].

Different parts (leaves, rhizomes, and roots) of the $D$. esculentum were extracted with aqueous and alcoholic sol- vents to evaluate the antibacterial activity by using the disk diffusion method. Four bacterial strains, namely, E. coli, Salmonella arizonae, Salmonella typhi, and Staphylococcus aureus, were used in this study. The rhizome and root extracts inhibited the growth of microorganisms while leaf extract did not show any inhibition. Additionally, extracts 
Table 3: Mineral composition of Diplazium esculentum from different regions.

\begin{tabular}{|c|c|c|c|c|c|c|c|c|c|c|}
\hline Parameters & $\begin{array}{c}\text { Bangladesh } \\
\text { (mg/100 g) } \\
{[32]}\end{array}$ & $\begin{array}{l}\text { Bangladesh } \\
\text { (mg/g) [34] }\end{array}$ & $\begin{array}{c}\begin{array}{c}\text { India } \\
(\mathrm{mg} / \\
100 \mathrm{~g}) \\
{[26]}\end{array} \\
\end{array}$ & $\begin{array}{c}\text { Indonesia } \\
(\mu \mathrm{g} / \mathrm{g}) \\
{[31]}\end{array}$ & $\begin{array}{c}\text { Malaysia } \\
(\mathrm{mg} / \mathrm{kg}) \\
{[28]}\end{array}$ & $\begin{array}{c}\text { India } \\
(\mathrm{mg} / \\
100 \mathrm{~g}) \\
{[57]}\end{array}$ & $\begin{array}{c}\text { India } \\
(\mathrm{mg} / \mathrm{g}) \\
{[25]}\end{array}$ & $\begin{array}{c}\text { Nepal } \\
(\mathrm{mg} / 100 \mathrm{~g}) \\
{[58]}\end{array}$ & $\begin{array}{c}\text { Indonesia } \\
(\mathrm{mg} / \mathrm{kg}) \\
{[27]}\end{array}$ & $\begin{array}{c}\text { India } \\
(\mathrm{mg} / \\
100 \mathrm{~g}) \\
{[59]}\end{array}$ \\
\hline $\bar{N}$ & - & 13.97 & - & - & - & - & & - & - & - \\
\hline $\mathrm{P}$ & 48 & 1.58 & - & - & - & - & & 117 & 0.09 & - \\
\hline K & - & 7.93 & - & - & - & 914.4 & & - & 0.24 & 927.4 \\
\hline $\mathrm{Ca}$ & 9 & - & 0.66 & - & - & 192.7 & & - & 0.39 & 200.5 \\
\hline $\mathrm{Mg}$ & 11 & - & 9.56 & - & - & 0.36 & $\begin{array}{c}10- \\
12.11\end{array}$ & - & 0.14 & - \\
\hline $\mathrm{Fe}$ & - & - & 14.38 & 15.7 & - & 11.2 & $\begin{array}{l}20.2- \\
23.4\end{array}$ & 1.03 & 44.6 & - \\
\hline $\mathrm{Mn}$ & - & - & 11.91 & 7.03 & $3.24-22.5$ & - & $\begin{array}{c}0.04- \\
0.38\end{array}$ & - & - & - \\
\hline $\mathrm{Na}$ & 54 & 20.21 & 0.50 & - & - & 9.5 & & - & - & 8.1 \\
\hline $\mathrm{Cu}$ & - & - & 13.37 & 3.99 & $3.24-24.3$ & 0.32 & $\begin{array}{l}1.03- \\
1.28\end{array}$ & - & 4.24 & - \\
\hline $\mathrm{Al}$ & - & - & 58.5 & 18.3 & - & - & $\begin{array}{c}0.10- \\
0.73\end{array}$ & - & - & - \\
\hline As & - & - & 14.6 & - & - & - & - & - & - & - \\
\hline $\mathrm{Cd}$ & - & - & 0.4 & - & - & - & - & - & - & - \\
\hline $\mathrm{Hg}$ & - & - & 0.07 & - & - & - & - & - & - & - \\
\hline $\mathrm{Li}$ & - & - & 2.1 & - & - & - & - & - & - & - \\
\hline $\mathrm{Ni}$ & - & - & 24.5 & - & - & - & - & - & - & - \\
\hline $\mathrm{Pb}$ & - & - & 0.8 & 2.46 & $0.31-3.26$ & - & - & - & - & - \\
\hline $\mathrm{Cr}$ & - & - & - & 0.05 & $1.19-3.03$ & - & - & - & - & - \\
\hline
\end{tabular}

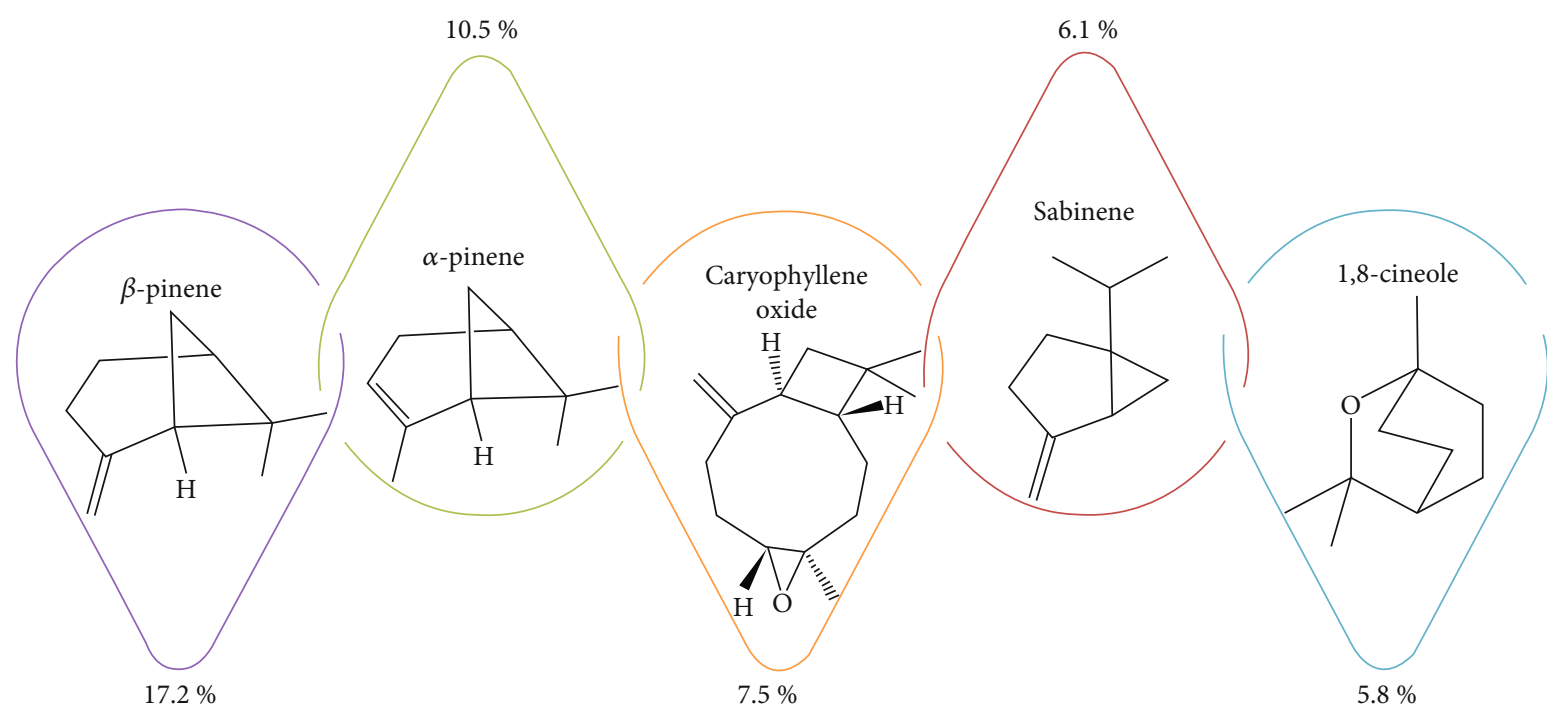

Figure 2: Main essential oil components of Diplazium esculentum [16].

combined with the antibiotic (tetracycline in equal amount) were more potent against bacterial strains than the antibiotic alone [96].

The aerial parts of D. esculentum extracts were evaluated for antimicrobial activity by using a colorimetric broth microdilution method. A total six different solvent extracts (hexane, chloroform, ethyl acetate, ethanol, methanol, and distilled water) were used against a series of microbial strains including S. aureus, B. cereus, Klebsiella pneumoniae, Pseudomonas aeruginosa, E. coli, Acinetobacter baumannii, Candida albicans, Candida parapsilosis, Issatchenkia orientalis, Cryptococcus neoformans, Aspergillus brasiliensis, and 
<smiles>O=C1OC([C@@H](O)CO)C(O)=C1O</smiles>

Ascorbic acid<smiles>Cc1c(C)c2c(c(C)c1O)CCC(C)(CCC[C@H](C)CCC[C@H](C)CCCC(C)C)O2</smiles>

Alpha-tocopherol<smiles>Cc1cc2c(c(C)c1CCO)C(=O)C(C)C2</smiles>

Pterosin-B<smiles>CC1=CCC(O)(C(C)C)CC1</smiles>

Terpene<smiles>O=C(O)/C=C/c1ccccc1</smiles>

Trans-cinnamic acid<smiles>CC1=C[C@@]2(O[C@@H]3O[C@H](CO)[C@@H](O)[C@H](O)[C@H]3O)C[C@H](C)C(=O)C2[C@@](C)(O)C12CC2</smiles>

Ptaquiloside<smiles>O=C(O)c1ccc(O)c(O)c1</smiles>

Protocatechuic acid<smiles>C=C(O)CCCCCCCCCCCCC</smiles><smiles>CC1=CC(O)CC(C)(C)C1/C=C/C(C)=C/C=C/C(C)=C/C=C/C/C=C(C)/C=C/C=C/C1=C(C)CC(O)CC1(C)C</smiles>

(a)<smiles>C=CC(=C)CCCC(C)CCCC(C)CCCC(C)C</smiles>

$\alpha$-Linolenic acid<smiles>CCCCCCCCCCCCCCCC(=O)OC</smiles>

Methyl palmitate<smiles>O=C(O)C(O)Cc1ccc(O)cc1</smiles>

3-(4-hydroxyphenyl)lactic acid<smiles>O=c1c(O)c(-c2ccc(O)c(O)c2)oc2cc(O)cc(O)c12</smiles><smiles>O=P(O)(O)O[C@H]1[C@H](OP(=O)(O)O)[C@@H](OP(=O)(O)O)[C@H](OP(=O)(O)O)[C@@H](OP(=O)(O)O)[C@H]1OP(=O)(O)O</smiles>

$\mathrm{OH}$

Phytate<smiles>CC(C)COC(=O)c1ccccc1C(=O)OCC(C)C</smiles>

Diisobutyl phthalate<smiles>CC[C@H](C[C@@H](O)C(C)(O)[C@H]1CC[C@]2(O)C3=CC[C@@H]4C[C@H](O)[C@@H](O)C[C@]4(C)[C@H]3CC[C@]12C)C(C)(C)O</smiles>

O Makisterone $\mathrm{C}$

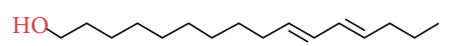

10,12-hexadecadien-1-ol<smiles>C[C@@H]1OC(OCC2O[C@@H](Oc3c(-c4ccc(O)c(O)c4)oc4cc(O)cc(O)c4c3=O)[C@H](O)[C@H](O)[C@H]2O)[C@H](O)[C@@H](O)[C@@H]1O</smiles><smiles>C=CC(=O)Nc1ccc(C#CC2(O)CCN(C)C2=O)cc1</smiles>

Amarasterone A<smiles>[R10][R16](=O)[O-]</smiles><smiles>C/C(=C\CO)CCC[C@H](C)CCC[C@H](C)CCCC(C)C</smiles>

Phytol

(b)

FIgUre 3: (a) Main nonoil bioactive components of D. esculentum. (b) Main nonoil bioactive components of D. esculentum.

Trichophyton mentagrophytes. The plant extract only showed a good-moderate antimicrobial activity against $I$. orientalis [97].

The methanolic extract of $D$. esculentum leaves has been evaluated for antibacterial activity by using the disc diffusion method [98]. The plant extract showed slight antibacterial activity (6-10 $\mathrm{mm}$ zone of inhibition) against Salmonella paratyphi, Vibrio parahaemolyticus, E. coli, B. megaterium,
Shigella dysenteriae, and Shigella boydii among 12 bacterial strains.

The chloroform and methanolic extracts of $D$. esculentum leaves were evaluated for antimicrobial activity by using the disk diffusion method [46]. The plant extracts showed inactive antimicrobial activity against all the microbial strains tested, namely, K. pneumoniae, S. aureus, E. coli, Salmonella typhimurium, Vibrio cholerae, Sarcina lutea, 


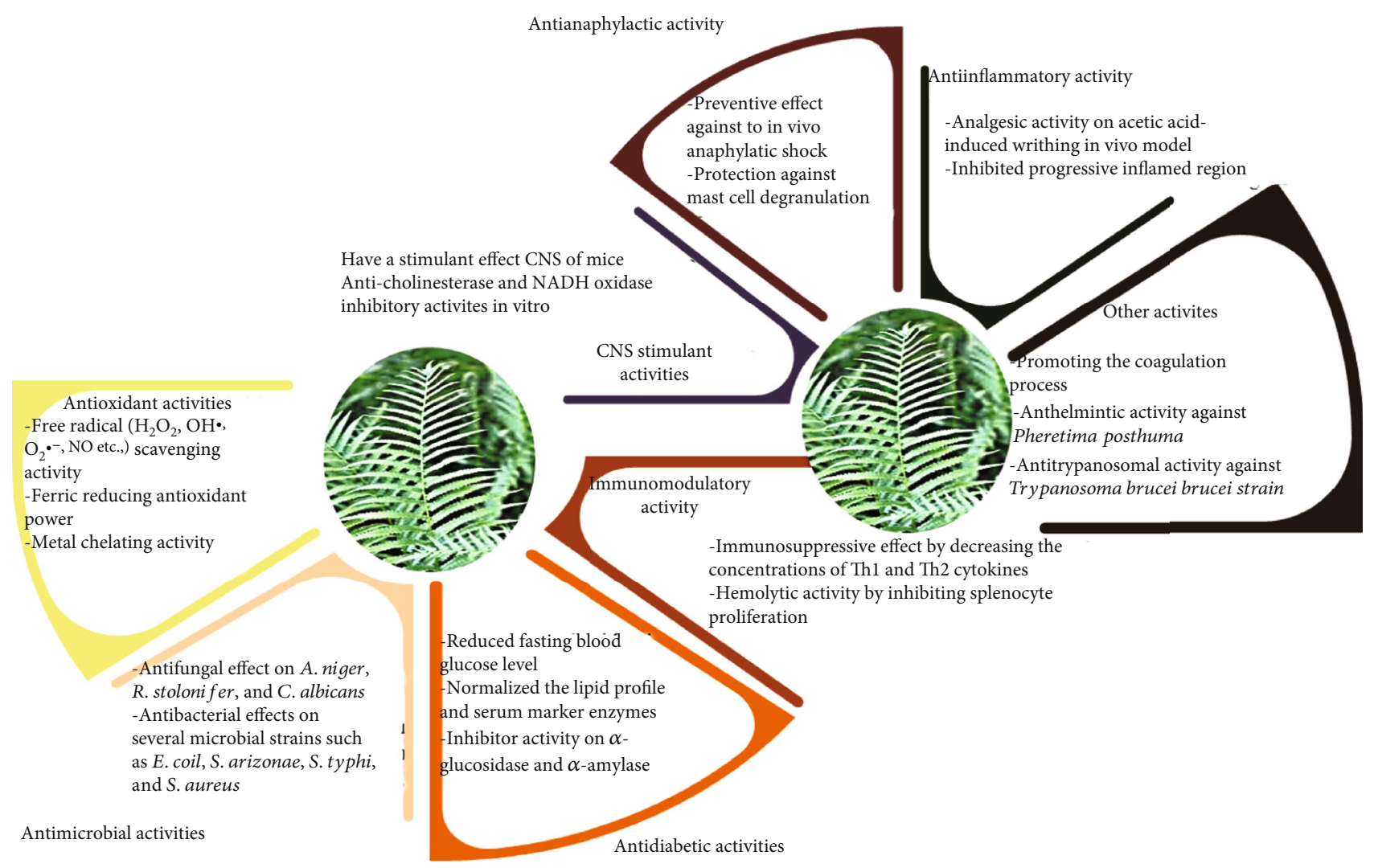

FIGURE 4: Summary of the proposed biological activities of Diplazium esculentum.

Bacillus subtilis, and Shigella boydii in terms of MIC (1.6$12.5 \mathrm{mg} / \mathrm{mL}$ ) value.

The antifungal activity of $D$. esculentum leaves against three fungal strains using the agar diffusion method has been reported [94]. The methanolic extract showed inactive antifungal activity against Aspergillus niger, Rhizopus stolonifer, and C. albicans in terms of MIC $(50-100 \mathrm{mg} / \mathrm{mL})$ and minimum fungal inhibition concentration $(100-200 \mathrm{mg} / \mathrm{mL})$.

4.3. Antidiabetic Activities. Diabetes mellitus is a chronic carbohydrate, fat, and protein metabolism disorder characterized by the increase in blood glucose level due to defect of insulin secretion [99]. The inhibition of $\alpha$-glucosidase and $\alpha$-amylase enzymes, involved in the digestion of carbohydrates, can significantly reduce the postprandial increase of blood glucose and therefore can be an important strategy in the management of blood glucose level in type 2 diabetic and borderline patients. The antidiabetic activity of D. esculentum through inhibition of $\alpha$-glucosidase and $\alpha$-amylase enzymes has been reported [69]. The results demonstrated that $D$. esculentum extract exhibited the highest $\alpha$-amylase (92.09\%) and $\alpha$-glucosidase (70.01\%) inhibitory activities.

The protective effect of a hydro-alcoholic extract of $D$. esculentum on streptozocin- (STZ-) induced diabetes was evaluated [82]. In this study, a total of 30 rats were used and treated with plant extract up to 21 days. After the treatment, it was observed that the plant extract $(500 \mathrm{mg} / \mathrm{kg})$ reduced $(50.2 \%)$ the blood glucose level in STZ-induced diabetic rats. Additionally, a significant reduction was recorded in plant extract-treated rats for lipid profiling $(p<0.01)$, serum marker enzyme activity $(p<0.001)$, necrosis, and regeneration of beta cells. The plant extract showed dosedependent activity in all the experiments.

4.4. Immunomodulatory Activity. The immunosuppressive and hemolytic activities of $D$. esculentum extracts in mouse models have been evaluated [100]. A total of 120 Swiss albino mice (6-8 weeks age) were treated with plant extracts up to 180 days. After this treatment, the plant extract showed significant dose-dependent decreases in body weight, relative spleen weight, number of plaques (formation of antibody secreting cells) formed, hemagglutination antibody titer value, the number of peritoneal macrophages, and the number of cultured splenocytes. The in vitro analysis showed significant dose-dependent increases in the percentage inhibition of splenocyte proliferation as well as the percentage of hemolysis. In other words, the treatment with $D$. esculentum may act as an immunosuppressive agent.

The impact of boiled D. esculentum on Th1 and Th2 cytokine levels of Swiss albino mice that were treated with different doses of plant extract, daily up to 180 days, has been reported [101]. The outcome of the study demonstrated that the plant extract significantly decreases the concentration of Th1 and Th2 cytokines when compared with controls. In other words, boiled $D$. esculentum extract may affect some of the innate and cell-mediated immune responses by modulating the level of Th1 and Th2 cytokines. 
Table 4: Previous antioxidant studies in Diplazium esculentum.

\begin{tabular}{|c|c|c|c|c|c|}
\hline \multirow[b]{2}{*}{ Plant part used and solvent system } & \multirow[b]{2}{*}{ Name of assay } & \multicolumn{3}{|c|}{ Key results } & \multirow[b]{2}{*}{ References } \\
\hline & & Plant extracts & Positive control & $\begin{array}{l}\text { Antioxidant } \\
\text { activity* }\end{array}$ & \\
\hline \multirow{3}{*}{$\begin{array}{l}\text { Whole plant, (chloroform, n-butanol, } \\
\text { aqueous) }\end{array}$} & $\begin{array}{c}\text { Free radical scavenging } \\
(\mathrm{DPPH})\end{array}$ & $\begin{array}{c}\mathrm{IC}_{50}=287 \\
-404 \mu \mathrm{g} / \mathrm{mL}\end{array}$ & $\begin{array}{c}\mathrm{IC}_{50}=17.45 \\
\mu \mathrm{g} / \mathrm{mL}\end{array}$ & Moderate & \multirow{3}{*}[77]{} \\
\hline & $\begin{array}{l}\text { Radical cation scavenging } \\
\text { activity }\left(\mathrm{ABTS}^{+}\right)\end{array}$ & $\begin{array}{c}\mathrm{IC}_{50}=191 \\
-273 \mu \mathrm{g} / \mathrm{mL}\end{array}$ & $\begin{array}{c}\mathrm{IC}_{50}=08.44 \\
\mu \mathrm{g} / \mathrm{mL}\end{array}$ & Moderate & \\
\hline & $\begin{array}{l}\text { Ferric reducing antioxidant } \\
\text { power (FRAP) }\end{array}$ & $0.44-0.55 \mathrm{mg} / \mathrm{g}$ & - & & \\
\hline Leaves (methanol) & $\begin{array}{l}\text { Free radical scavenging } \\
(\mathrm{DPPH})\end{array}$ & $\begin{array}{l}31.35-57.95 \% \\
\text { inhibition }\end{array}$ & $\begin{array}{l}\text { 91.99-97.03\% } \\
\text { inhibition }\end{array}$ & Moderate & {$[57]$} \\
\hline Leaves (methanol) & $\begin{array}{l}\text { Free radical scavenging } \\
(\mathrm{DPPH})\end{array}$ & $\begin{array}{c}\mathrm{IC}_{50}=402.88 \mu \mathrm{g} / \\
\mathrm{mL}\end{array}$ & $\begin{array}{c}\mathrm{IC}_{50}=324.86 \\
\mu \mathrm{g} / \mathrm{mL}\end{array}$ & Weak & {$[83]$} \\
\hline \multirow{3}{*}{ Leaves (protein) } & $\begin{array}{l}\text { Free radical scavenging } \\
(\mathrm{DPPH})\end{array}$ & $\begin{aligned} & \mathrm{IC}_{50}= 10.23 \mathrm{mg} / \\
& \mathrm{mL}\end{aligned}$ & - & - & \multirow{3}{*}[84]{} \\
\hline & $\begin{array}{l}\text { Free radical scavenging } \\
(\mathrm{DMPD}+)^{-}\end{array}$ & $\begin{aligned} \mathrm{IC}_{50}= & 14.67 \mathrm{mg} / \\
& \mathrm{mL}\end{aligned}$ & - & - & \\
\hline & $\begin{array}{l}\text { Radical cation scavenging } \\
\text { activity }\left(\mathrm{ABTS}^{+}\right)\end{array}$ & $\begin{aligned} & \mathrm{IC}_{50}= 07.95 \mathrm{mg} / \\
& \mathrm{mL}\end{aligned}$ & - & - & \\
\hline Leaves (not reported) & $\begin{array}{l}\text { Free radical scavenging } \\
(\mathrm{DPPH})\end{array}$ & $\begin{array}{l}\text { 336-3359 ORAC } \\
\text { unit }^{2} / g\end{array}$ & - & - & {$[50]$} \\
\hline $\begin{array}{l}\text { Leaves (ethanol, vinegar, acetic acid, } \\
\text { aqueous) }\end{array}$ & $\begin{array}{l}\text { Free radical scavenging } \\
\text { activity (DPPH) }\end{array}$ & $\begin{array}{c}258-303 \mu \mathrm{mol} \\
\mathrm{TE} / 100 \mathrm{~g}\end{array}$ & - & - & {$[85]$} \\
\hline \multirow{2}{*}{ Leaves (chloroform, methanol) } & $\begin{array}{l}\text { Total antioxidant capacities } \\
\text { (TAC) }\end{array}$ & $\begin{array}{c}181.94- \\
207.41 \mathrm{mg} / \mathrm{g}\end{array}$ & - & - & \multirow{2}{*}[46]{} \\
\hline & $\begin{array}{l}\text { Free radical scavenging } \\
(\mathrm{DPPH})\end{array}$ & $\begin{array}{c}\mathrm{IC}_{50}=5907 \\
-95669 \mu \mathrm{g} / \mathrm{mL}\end{array}$ & $\begin{array}{c}\mathrm{IC}_{50}=13.76 \\
\mu \mathrm{g} / \mathrm{mL}\end{array}$ & Weak & \\
\hline \multirow{4}{*}{ Leaves (methanol) } & $\begin{array}{l}\text { Free radical scavenging } \\
\text { activity }(\mathrm{DPPH})\end{array}$ & $\begin{array}{c}\mathrm{IC}_{50}=1.73 \mathrm{mg} / \\
\mathrm{mL}\end{array}$ & - & - & \multirow{4}{*}[86]{} \\
\hline & Metal chelating activity & $52.07 \mathrm{mg} / \mathrm{mL}$ & - & - & \\
\hline & $\begin{array}{c}\text { Ferric reducing antioxidant } \\
\text { power (FRAP) }\end{array}$ & $2.12 \mu \mathrm{g} / \mathrm{mg}$ & - & - & \\
\hline & $\begin{array}{l}\text { Radical cation scavenging } \\
\text { activity }\left(\mathrm{ABTS}^{+}\right)\end{array}$ & $\begin{array}{c}\mathrm{IC}_{50}=0.03 \mathrm{mg} / \\
\mathrm{mL}\end{array}$ & - & - & \\
\hline \multirow{2}{*}{ Fronds (aqueous, ethanol) } & $\begin{array}{l}\text { Radical cation scavenging } \\
\text { activity }\left(\mathrm{ABTS}^{+}\right)\end{array}$ & $\begin{array}{l}09.60-57.84 \% \\
\text { inhibition }\end{array}$ & - & - & \multirow{2}{*}[65]{} \\
\hline & $\begin{array}{l}\text { Hydrogen peroxide } \\
\text { scavenging }\left(\mathrm{H}_{2} \mathrm{O}_{2}\right)\end{array}$ & $\begin{array}{c}15-40 \% \\
\text { inhibition }\end{array}$ & $50 \%$ inhibition & Strong & \\
\hline \multirow{7}{*}{ Leaves (methanol) } & $\begin{array}{l}\text { Hydroxyl radical } \\
\text { scavenging }(\mathrm{OH} \cdot)\end{array}$ & $\begin{aligned} & \mathrm{IC}_{50}= 811.00 \mu \mathrm{g} / \\
& \mathrm{mL}\end{aligned}$ & $\begin{array}{c}\mathrm{IC}_{50}=571.00 \\
\mu \mathrm{g} / \mathrm{mL}\end{array}$ & Weak & \multirow{7}{*}[87]{} \\
\hline & $\begin{array}{l}\text { Superoxide anion } \\
\text { scavenging }\left(\mathrm{O}_{2} \cdot{ }^{-}\right)\end{array}$ & $\begin{array}{c}\mathrm{IC}_{50}=90.39 \mu \mathrm{g} / \\
\mathrm{mL}\end{array}$ & $\begin{array}{c}\mathrm{IC}_{50}=42.06 \\
\mu \mathrm{g} / \mathrm{mL}\end{array}$ & Strong & \\
\hline & $\begin{array}{l}\text { Nitric oxide radical } \\
\text { scavenging }(\mathrm{NO})\end{array}$ & $\begin{aligned} & \mathrm{IC}_{50}= 204.28 \mu \mathrm{g} / \\
& \mathrm{mL}\end{aligned}$ & $\begin{array}{c}\mathrm{IC}_{50}=90.82 \\
\mu \mathrm{g} / \mathrm{mL}\end{array}$ & Moderate & \\
\hline & $\begin{array}{l}\text { Hydrogen peroxide } \\
\text { scavenging }\left(\mathrm{H}_{2} \mathrm{O}_{2}\right)\end{array}$ & $\begin{array}{c}\mathrm{IC}_{50}=4.17 \mathrm{mg} / \\
\mathrm{mL}\end{array}$ & $\begin{array}{c}\mathrm{IC}_{50}=3.24 \mu \mathrm{g} / \\
\mathrm{mL}\end{array}$ & Strong & \\
\hline & $\begin{array}{c}\text { Peroxynitrite scavenging } \\
\left(\mathrm{ONOO}^{-}\right)\end{array}$ & $\begin{array}{c}\mathrm{IC}_{50}=3.35 \mathrm{mg} / \\
\mathrm{mL}\end{array}$ & $\begin{array}{c}\mathrm{IC}_{50}=0.87 \mu \mathrm{g} / \\
\mathrm{mL}\end{array}$ & Strong & \\
\hline & $\begin{array}{l}\text { Singlet oxygen scavenging } \\
\qquad\left({ }^{1} \mathrm{O}_{2}\right)\end{array}$ & $\begin{aligned} & \mathrm{IC}_{50}= 278.88 \mu \mathrm{g} / \\
& \mathrm{mL}\end{aligned}$ & $\begin{array}{c}\mathrm{IC}_{50}=46.15 \\
\mu \mathrm{g} / \mathrm{mL}\end{array}$ & Moderate & \\
\hline & $\begin{array}{l}\text { Hypochlorous acid } \\
\text { scavenging (HOCl) }\end{array}$ & $\begin{aligned} & \mathrm{IC}_{50}= 338.96 \mu \mathrm{g} / \\
& \mathrm{mL}\end{aligned}$ & $\begin{array}{c}\mathrm{IC}_{50}=235.95 \\
\mu \mathrm{g} / \mathrm{mL}\end{array}$ & Moderate & \\
\hline
\end{tabular}


TABLE 4: Continued.

\begin{tabular}{|c|c|c|c|c|c|}
\hline \multirow[b]{2}{*}{ Plant part used and solvent system } & \multirow[b]{2}{*}{ Name of assay } & \multicolumn{3}{|c|}{ Key results } & \multirow[b]{2}{*}{ References } \\
\hline & & Plant extracts & Positive control & $\begin{array}{l}\text { Antioxidant } \\
\text { activity }^{*}\end{array}$ & \\
\hline & Iron chelating & $\begin{array}{c}\mathrm{IC}_{50}=1.33 \mathrm{mg} / \\
\mathrm{mL}\end{array}$ & $\begin{array}{c}\mathrm{IC}_{50}=0.001 \\
\mu \mathrm{g} / \mathrm{mL}\end{array}$ & Strong & \\
\hline & $\begin{array}{l}\text { Lipid peroxidation } \\
\text { inhibition }\end{array}$ & $\begin{aligned} & \mathrm{IC}_{50}= 141.67 \mu \mathrm{g} / \\
& \mathrm{mL}\end{aligned}$ & $\begin{array}{c}\mathrm{IC}_{50}=6.76 \mu \mathrm{g} / \\
\mathrm{mL}\end{array}$ & Moderate & \\
\hline $\begin{array}{l}\text { Leaves (petroleum ether, chloroform, } \\
\text { acetone, methanol, aqueous) }\end{array}$ & $\begin{array}{l}\text { Ferric reducing antioxidant } \\
\text { power (FRAP) }\end{array}$ & $\begin{array}{l}0.22-7.6 \mathrm{mM} / \mathrm{dry} \\
\text { weight }\end{array}$ & - & - & {$[88]$} \\
\hline \multirow{2}{*}{ Leaves (aqueous-methanol, acetone) } & $\begin{array}{l}\text { Free radical scavenging } \\
(\mathrm{DPPH})\end{array}$ & $\begin{array}{c}\mathrm{IC}_{50}=0.92 \\
-3.60 \mathrm{mg} \text { dry wt. }\end{array}$ & - & - & \multirow{2}{*}[89]{} \\
\hline & $\begin{array}{l}\text { Ferric reducing antioxidant } \\
\text { power (FRAP) }\end{array}$ & $4.99-8.78 \mathrm{mg} / \mathrm{g}$ & - & - & \\
\hline Leaves (methanol) & $\begin{array}{l}\text { Free radical scavenging } \\
(\mathrm{DPPH})\end{array}$ & $\begin{aligned} \mathrm{EC}_{50}= & 3353.2 \mu \mathrm{g} / \\
& \mathrm{mg}\end{aligned}$ & $\begin{array}{c}\mathrm{EC}_{50}=322.4 \\
\mu \mathrm{g} / \mathrm{mg}\end{array}$ & Weak & {$[90]$} \\
\hline \multirow{3}{*}{ Leaves (aqueous) } & $\begin{array}{l}\text { Free radical scavenging } \\
(\mathrm{DPPH})\end{array}$ & $50 \mu \mathrm{mol} / \mathrm{g}$ & - & - & \multirow{3}{*}[91]{} \\
\hline & $\begin{array}{l}\text { Ferric reducing antioxidant } \\
\text { power (FRAP) }\end{array}$ & $100 \mathrm{~mol} / \mathrm{g}$ & - & - & \\
\hline & Cupric ions chelation assay & $80 \%$ inhibition & - & - & \\
\hline
\end{tabular}

4.5. CNS Stimulant Activities. The impact of "Ulam" (a fresh Malaysian vegetable, $D$. esculentum) on cognitive status has been evaluated [102]. In this cross-sectional study, a total of 132 adults were recruited. Socio-demographic information, anthropometric measurements, dietary history, food frequency, and cognitive function were assessed. The average ulam intake by the participants was $15.1 \pm 8.2 \mathrm{~g} /$ day. The outcome of the study indicated that "pucukpaku" showed protective effects (62.9\%) against cognitive decline.

The anticholinesterase and NADH oxidase inhibitory activities of a methanolic extract of $D$. esculentum have been evaluated [83]. Recently, most of the studies reported that the inhibition of anticholinesterase has been shown to be a strategy for the treatment of neurodegenerative disorders. The results of the study demonstrated that the methanolic extract of $D$. esculentum inhibited acetyl-cholinesterase and $\mathrm{NADH}$ oxidase in a dose-dependent manner, with $\mathrm{IC}_{50}$ values of 272.97 and $265.81 \mu \mathrm{g} / \mathrm{mL}$.

The CNS stimulant effect of $D$. esculentum in a mouse model using digital acto-photometer has been reported [88]. The plant water extract showed statistically significant $(p<0.0001)$ and dose-dependent activity when compared with control and standard caffeine.

4.6. Toxicity Studies (In Vitro and In Vivo). The methanolic and chloroform extracts of D. esculentum were evaluated for their toxicity using brine shrimp lethality bioassay. Both extracts produced dose-dependent increment in percent mortality of brine shrimp nauplii which indicates the presence of toxic compounds in the extracts. The $\mathrm{LC}_{50}$ values were recorded as $1.87 \mu \mathrm{g} / \mathrm{mL}$ (chloroform), $1.62 \mu \mathrm{g} / \mathrm{mL}$ (methanol), and $0.66 \mu \mathrm{g} / \mathrm{mL}$ (vincristine sulphate as standard drug) [46]. In another study, the toxicity of methanolic extract of $D$. esculentum using brine shrimp lethality bioassay was reported as significant $\left(\mathrm{LC}_{50}=18.6 \mu \mathrm{g} / \mathrm{mL}\right)$. [98]. In other study, the cytotoxicity of ethanolic extract of $D$. esculentum was evaluated in different cell lines including breast cancer (MDA-MB-231 and MCF-7), colon cancer (Caco-2), liver cancer (HepG2), and normal liver (Chang liver), and no cytotoxic effect was observed [103].

The systemic toxicity and several pathological effects of D. esculentum were evaluated on rabbits and guinea pigs [104]. The study indicated that the plant extract decreased all the pathological functions including growth, body weight, forced motor activity, alterations of blood glucose values, erythrocyte sedimentation rate, mean corpuscular volume, mean corpuscular hemoglobin, total leukocyte count, neutrophil, lymphocyte, and monocyte count, while increased blood SGOT level in both rats and guinea pigs. In other words, the plant extract indicated toxic effects in guinea pigs and rabbits, while rats showed a little adverse effects. Junejo and coworkers reported the nontoxic effects of $D$. esculentum extract on experimental models and recommended as a potential functional food [67].

The toxicological impact of $D$. esculentum on male reproductive functions of Swiss albino mice has been reported [105]. A total of 120 male Swiss albino mice of 6-8 weeks of age were fed orally with 80,160 , and $320 \mathrm{mg} / \mathrm{kg}$ b.w. of plant material and treated up to 180 days. After this successful treatment, the boiled plant extract showed significant dose and time-dependent decreases in body weight, absolute and relative testis 
TABle 5: Antimicrobial activities of Diplazium esculentum.

\begin{tabular}{|c|c|c|c|c|c|}
\hline Plant part used and solvent system & Microorganism & $\begin{array}{l}\text { Antimicrobial } \\
\text { activity }\end{array}$ & $\begin{array}{l}\mathrm{MIC}(\mu \mathrm{g} / \\
\mathrm{mL})\end{array}$ & $\begin{array}{l}\text { MBC or MFC }(\mu \mathrm{g} / \\
\mathrm{mL})\end{array}$ & Reference \\
\hline Aerial parts (ethanol) & $\begin{array}{c}\text { Bacillus cereus } \\
\text { Escherichia coli } \\
\text { Aspergillus ochraceus } \\
\text { Bacillus megaterium }\end{array}$ & $\begin{array}{l}\text { Moderate } \\
\text { Moderate } \\
\text { Moderate } \\
\text { Weak }\end{array}$ & $\begin{array}{l}200 \\
400 \\
400 \\
800\end{array}$ & $\begin{array}{c}800 \\
>800 \\
800 \\
>800\end{array}$ & {$[95]$} \\
\hline Aerial parts (ethanol) & $\begin{array}{c}\text { Staphylococcus aureus } \\
\text { Bacillus cereus } \\
\text { Klebsiella pneumoniae } \\
\text { Pseudomonas aeruginosa } \\
\text { Candida albicans } \\
\text { Candida parapsilosis } \\
\text { Cryptococcus neoformans } \\
\text { Issatchenkia orientalis }\end{array}$ & $\begin{array}{c}\text { Moderate-weak } \\
\text { Moderate-weak } \\
\text { Moderate-weak } \\
\text { Weak } \\
\text { Weak-inactive } \\
\text { Weak-inactive } \\
\text { Moderate } \\
\text { Good-moderate }\end{array}$ & $\begin{array}{c}310-630 \\
310-630 \\
310-630 \\
630 \\
630-1250 \\
1250-2500 \\
310 \\
80-160\end{array}$ & $\begin{array}{l}\text { NA } \\
1250 \\
\text { NA } \\
\text { NA } \\
2500 \\
\text { NA } \\
310 \\
160\end{array}$ & {$[97]$} \\
\hline Aerial parts (hexane) & $\begin{array}{l}\text { Cryptococcus neoformans } \\
\text { Issatchenkia orientalis }\end{array}$ & $\begin{array}{l}\text { Moderate } \\
\text { Good }\end{array}$ & $\begin{array}{c}310 \\
80 \\
\end{array}$ & $\begin{array}{l}310 \\
160\end{array}$ & {$[97]$} \\
\hline Aerial parts (chloroform) & $\begin{array}{c}\text { Staphylococcus aureus } \\
\text { Bacillus cereus } \\
\text { Klebsiella pneumoniae } \\
\text { Pseudomonas aeruginosa } \\
\text { Trichophyton } \\
\text { mentagrophytes } \\
\text { Candida albicans } \\
\text { Cryptococcus neoformans } \\
\text { Issatchenkia orientalis }\end{array}$ & $\begin{array}{c}\text { Moderate-weak } \\
\text { Moderate } \\
\text { Moderate-weak } \\
\text { Weak-inactive } \\
\text { Weak-inactive } \\
\text { Weak-inactive } \\
\text { Moderate } \\
\text { Good }\end{array}$ & $\begin{array}{c}310-630 \\
310 \\
310-630 \\
630-1250 \\
630-1250 \\
630-1250 \\
310 \\
80\end{array}$ & $\begin{array}{l}\text { NA } \\
630 \\
\text { NA } \\
\text { NA } \\
1250 \\
\text { NA } \\
310 \\
160\end{array}$ & [97] \\
\hline Aerial parts (ethyl acetate) & $\begin{array}{l}\text { Pseudomonas aeruginosa } \\
\text { Cryptococcus neoformans } \\
\text { Issatchenkia orientalis }\end{array}$ & $\begin{array}{l}\text { Weak } \\
\text { Moderate } \\
\text { Moderate }\end{array}$ & $\begin{array}{l}630 \\
310 \\
160 \\
\end{array}$ & $\begin{array}{l}\text { NA } \\
310 \\
310 \\
\end{array}$ & {$[97]$} \\
\hline Aerial parts (methanol) & $\begin{array}{c}\text { Staphylococcus aureus } \\
\text { Bacillus cereus } \\
\text { Klebsiella pneumoniae } \\
\text { Pseudomonas aeruginosa } \\
\text { Cryptococcus neoformans } \\
\text { Issatchenkia orientalis }\end{array}$ & $\begin{array}{l}\text { Weak-inactive } \\
\text { Weak-inactive } \\
\text { Weak-inactive } \\
\text { Weak-inactive } \\
\text { Moderate } \\
\text { Moderate } \\
\end{array}$ & $\begin{array}{c}630-1250 \\
630-1250 \\
630-1250 \\
630-1250 \\
310 \\
160 \\
\end{array}$ & $\begin{array}{c}\text { NA } \\
2500 \\
\text { NA } \\
\text { NA } \\
310 \\
310 \\
\end{array}$ & [97] \\
\hline Aerial parts (aqueous) & $\begin{array}{l}\text { Pseudomonas aeruginosa } \\
\text { Cryptococcus neoformans } \\
\text { Issatchenkia orientalis }\end{array}$ & $\begin{array}{l}\text { Weak-inactive } \\
\text { Moderate } \\
\text { Moderate }\end{array}$ & $\begin{array}{c}630-1250 \\
160-310 \\
160\end{array}$ & $\begin{array}{l}\text { NA } \\
310 \\
310\end{array}$ & [97] \\
\hline $\begin{array}{l}\text { Leaves, rhizomes, and roots (aqueous and } \\
\text { alcoholic) }\end{array}$ & $\begin{array}{c}\text { Escherichia coli } \\
\text { Salmonella arizonae } \\
\text { Salmonella typhi } \\
\text { Staphylococcus aureus }\end{array}$ & & $\begin{array}{l}\text {-* }^{*} \\
\text {-* }^{*} \\
\text {-* }^{*}\end{array}$ & & {$[96]$} \\
\hline Leaves (methanol) & $\begin{array}{l}\text { Salmonella paratyphi } \\
\text { Vibrio parahemolyticus } \\
\text { Escherichia coli } \\
\text { Bacillus megaterium } \\
\text { Shigella dysenteriae } \\
\text { Shigella boydii }\end{array}$ & & $\begin{array}{l}-^{*} \\
-^{*} \\
-* \\
-* \\
-^{*} \\
-*\end{array}$ & & {$[98]$} \\
\hline
\end{tabular}

MBC: minimum bactericidal concentration; MIC: minimum inhibitory concentration; MFC: minimum fungal concentration; NA: no activity; “_”: not tested. Antimicrobial activity was considered good (MIC less than $100 \mu \mathrm{g} / \mathrm{mL}$ ), moderate (MIC from 100 to $500 \mu \mathrm{g} / \mathrm{mL}$ ), and weak (MIC from 500 to $1000 \mu \mathrm{g} / \mathrm{mL}$ ). * Only zone inhibition test was performed.

weight, the relative weight of other organs and their biochemical parameters, percentage of live spermatozoa, fertility, and fecundity in plant extract fed mice. In other words, the main outcome of this study is boiled extracts of D. esculentum possess toxic properties that can be slow down the male reproductive functions and may induce infertility.

4.7. Antianaphylactic and Mast Cells Stabilizing Activity. D. esculentum were extracted with aqueous and ethanolic 
solvents and evaluated for mast cell stability and antianaphylactic activity. In this study, Swiss albino mice (18-20 g) and Wistar rats (150-170 g) were used. A significant reduction was observed in the number of degranulated mast cells of the plant extracts-treated models $(p<0.001)$. After the administration of both extracts at 250 and $500 \mathrm{mg} / \mathrm{kg}$ doses, it showed $72.83 \%, 76.67 \%, 69 \%$, and $71.67 \%$ intact mast cells. Plant extract demonstrates protective activity against mast cell degranulation. The $500 \mathrm{mg} / \mathrm{kg}$ dose of both extracts showed maximum inhibition of the release of myeloperoxidase from lung tissue. Additionally, the plant extract had stabilized the mast cell membrane and decreased the level of nitric oxide in serum and peritoneal fluid [106].

4.8. Anti-inflammatory Activity. The ethanolic extract of $D$. esculentum was evaluated for anti-inflammatory activity [107]. A total of 25 male mice were recruited in this experiment and divided into 5 groups. The ethanolic extract indicated anti-inflammatory activity on hind paw oedema in terms of inflamed inhibition percent of $125 \mathrm{mg} / \mathrm{kg}$ b.w. (71.72\%), $250 \mathrm{mg} / \mathrm{kg}$ b.w. (81.49\%), and $250 \mathrm{mg} / \mathrm{kg}$ b.w. $(92.60 \%)$ in the treated group. In another study, a considerable analgesic activity of $D$. esculentum was recorded using the acetic acid-induced writhing method in mice [63].

4.9. Other Biological Activities. The aqueous and powder extract of $D$. esculentum leaves was evaluated for coagulant activity [108]. The plant extracts combined with polyaluminium chloride showed a synergistic effect for all the measured parameters in Kuala Sepetang Landfill Site (KSLS) leachates. The combination was identified as a high molecular weight polymer, and it acted as an anionic coagulant and was also capable of promoting the coagulation process.

Extracts of the rhizome of $D$. esculentum extracts were evaluated for their anthelmintic activity against Pheretima posthuma. The study included three solvents (ethanol, aqueous, and petroleum ether) and three concentrations (10, 25, and $50 \mathrm{mg} / \mathrm{mL}$ ), and all the extracts demonstrated significant anthelmintic activity in terms of the time of paralysis and time of death. Ethanolic extract showed the highest activity compared to other solvents, and the activity was recorded in dose-dependent patterns [109].

Silver nanoparticles $(10-45 \mathrm{~nm})$ were synthesized using the leaf powder of D. esculentum [110]. The synthesized nanoparticles were evaluated as a catalyst in the degradation of methylene blue and rhodamine B and also evaluated for the anticoagulation activity. The synthesized Ag NPs showed considerable anticoagulation activity. Besides, prominent photocatalytic activity in the degradation of methylene blue and rhodamine B was also recorded.

The antitrypanosomal activity of $D$. esculentum leaves was evaluated against Trypanosoma brucei brucei strain BS221 [111]. In this study, the ethanolic extract was used with seven different concentrations ( 0.01 to $12.5 \mu \mathrm{g} / \mathrm{mL}$ ), and the extract showed significant antitrypanosomal activity with $\mathrm{IC}_{50}$ value $4.32 \mu \mathrm{g} / \mathrm{mL}$ and a selectivity index (SI) value $>23$ in mammalian cell line (Vero, $\mathrm{IC}_{50}>100 \mu \mathrm{g} / \mathrm{mL}$ ) when compared with the positive control (pentamidine, I $\mathrm{C}_{50}=4.51 \mathrm{ng} / \mathrm{mL}$ ).

\section{Concluding Remark and Future Prospective}

The present manuscript reports traditional uses, nutraceuticals, pharmacognosy, phytochemistry, and pharmacological studies in D. esculentum. The literature survey revealed that D. esculentum is one of the most important and popular wild species of ferns in the Himalaya. It is a widely used species in different traditional systems, but the complete chemical composition and active compounds need to be further elucidated and authenticated by bioassay-guided isolation. However, very limited studies are available for this species, not only in terms of chemical characterization but also in terms of pharmacological evaluation as well. Most of the studies are limited to the in vitro screening and a few for in vivo. Clinical trial studies should be performed to evaluate the safety profile of wild ferns in the human body in terms of antimicrobial activity, antidiabetic activity, antiinflammatory activity, and immunomodulatory aspects. Apart from this, educating the local people regarding the cultivation, conservation, and sustainable utilization of this plant will help for improving the population size of the species.

\section{Data Availability}

The data used to support the findings of this study are available from the corresponding author upon request.

\section{Conflicts of Interest}

The authors declared that they have no conflict of interest regarding this manuscript.

\section{Acknowledgments}

Help and support received from the Graphic Era University, Dehradun, Uttarakhand, India, is duly acknowledged. P.S. thank Dr. Ashish Bahuguna for their help in drawing the chemical structure of bioactive compounds.

\section{References}

[1] M. E. Alonso-Amelot, A. Oliveros, and M. P. Calcagno-Pisarelli, "Phenolics and condensed tannins in relation to altitude in neotropical Pteridium spp: a field study in the Venezuelan Andes," Biochemical Systematics and Ecology, vol. 32, no. 11, pp. 969-981, 2004.

[2] U. Amna and V. Mardina, "Antioxidant activity of methanol extract of Diplazium esculentum (Retz.) Sw. leaves collected from Aceh," in IOP Conference Series: Materials Science and Engineering, Volume 725, 3rd Nommensen International Conference on Technology and Engineering 2019 (3rd NICTE), p. 012082, Indonesia, July 2019.

[3] P. Semwal, S. Painuli, D. Tewari, R. W. Bussmann, L. M. S. Palni, and A. Thapliyal, "Assesment of non-timber Brahma Kamal (Saussurea obvallata (DC.) Edgew.), an important Himalayan medicinal plant: Ethnomedicinal, phytochemical and pharmacological overview," Ethnobotany Research \& Applications, vol. 19, no. 40, 2020. 
[4] N. M. Zlatic and M. S. Stankovic, "Variability of secondary metabolites of the species Cichorium intybus L. from different habitats," Plants, vol. 6, no. 3, p. 38, 2017.

[5] Z. Bharucha and J. Pretty, "The roles and values of wild foods in agricultural systems," Philosophical Transactions of the Royal Society of London. Series B, Biological Sciences, vol. 365, no. 1554, pp. 2913-2926, 2010.

[6] R. K. Maikhuri, K. S. Rao, and K. G. Saxena, "Bioprospecting of wild edibles for rural development in the central Himalayan mountains of India," Mountain Research and Development, vol. 24, no. 2, pp. 110-113, 2004.

[7] M. Sharma, P. Dwivedi, A. Singh Rawat, and A. K. Dwivedi, "Nutrition nutraceuticals: a proactive approach for healthcare," Nutraceuticals, vol. 4, pp. 79-116, 2016.

[8] S. Konsam, B. Thongam, and A. K. Handique, "Assessment of wild leafy vegetables traditionally consumed by the ethnic communities of Manipur, northeast India," Journal of Ethnobiology and Ethnomedicine, vol. 12, no. 1, p. 9, 2016.

[9] T. Belwal, A. Pandey, I. D. Bhatt, R. S. Rawal, and Z. Luo, "Trends of polyphenolics and anthocyanins accumulation along ripening stages of wild edible fruits of Indian Himalayan region,” Scientific Reports, vol. 9, no. 1, p. 5894, 2019.

[10] S. Misra, R. Maikhuri, D. Dhyani, and K. Rao, "Assessment of traditional rights, local interference and natural resource management in Kedarnath Wildlife Sanctuary," International Journal of Sustainable Development \& World Ecology, vol. 16, no. 6, pp. 404-416, 2009.

[11] PPG I, "A community-derived classification for extant lycophytes and ferns," Journal of Systematics and Evolution, vol. 54, no. 6, pp. 563-603, 2016.

[12] V. Manickam and V. Irudayaraj, Pteridophyte flora of the Western Ghats, BI publications, South India, 1992.

[13] V. Manickam and V. Irudyaraj, Pteridophytic flora of Nilgiris South India, Bishen Singh Mahendra Pal Singh, Dehradun, 2003.

[14] U. Quattrocchi, CRC World Dictionary of Medicinal and Poisonous Plants: Common Names, Scientific Names, Eponyms, Synonyms, and Etymology (5 Volume Set), CRC press, 2016.

[15] R. Abe and K. Ohtani, "An ethnobotanical study of medicinal plants and traditional therapies on Batan Island, the Philippines," Journal of Ethnopharmacology, vol. 145, no. 2, pp. 554-565, 2013.

[16] E. Essien, R. Ascrizzi, and G. Flamini, "Characterization of volatile compounds of Diplazium esculentum," Chemistry of Natural Compounds, vol. 55, no. 5, pp. 958-959, 2019.

[17] M. F. Kadir, M. S. B. Sayeed, N. I. Setu, A. Mostafa, and M. Mia, "Ethnopharmacological survey of medicinal plants used by traditional health practitioners in Thanchi, Bandarban Hill Tracts, Bangladesh," Journal of Ethnopharmacology, vol. 155, no. 1, pp. 495-508, 2014.

[18] O. Lense, "Biological screening of selected traditional medicinal plants species utilized by local people of Manokwari, West Papua Province," Nusantara Bioscience, vol. 3, no. 3, 2016.

[19] K. Roosita, C. M. Kusharto, M. Sekiyama, Y. Fachrurozi, and R. Ohtsuka, "Medicinal plants used by the villagers of a Sundanese community in West Java, Indonesia," Journal of Ethnopharmacology, vol. 115, no. 1, pp. 72-81, 2008.

[20] H. Tag, P. Kalita, P. Dwivedi, A. Das, and N. D. Namsa, "Herbal medicines used in the treatment of diabetes mellitus in Arunachal Himalaya, northeast, India," Journal of Ethnopharmacology, vol. 141, no. 3, pp. 786-795, 2012.
[21] F. Zannah, M. Amin, H. Suwono, and B. Lukiati, "Phytochemical screening of Diplazium esculentum as medicinal plant from Central Kalimantan, Indonesia," AIP Conference Proceedings, vol. 1844, article 050001, 2017.

[22] B. Sarkar, M. Basak, M. Chowdhury, and A. Das, "Importance of Diplazium esculentum (Retz.) Sw. (Athyriaceae) on the lives of local ethnic communities in Terai and Duars of West Bengal-A report," Plant Archives, vol. 18, pp. 439-442, 2018.

[23] A. Kutum, R. Sarmah, and D. Hazarika, "An ethnobotanical study of Mishing tribe living in fringe villages of Kaziranga National Park of Assam, India," Indian Journal of Fundamental and Applied Life Sciences, vol. 1, pp. 45-61, 2011.

[24] E. C. Sousa, A. M. A. Uchôa-Thomaz, J. O. B. Carioca et al., "Chemical composition and bioactive compounds of grape pomace (Vitis vinifera L.), Benitaka variety, grown in the semiarid region of Northeast Brazil," Food Science and Technology, vol. 34, no. 1, pp. 135-142, 2014.

[25] G. N. Archana, S. Pradeesh, M. D. Chinmayee, I. Mini, and T. Swapna, "Diplazium esculentum: a wild nutrient-rich leafy vegetable from Western Ghats," in Prospects in Bioscience: Addressing the issues, pp. 293-301, Springer, 2012.

[26] S. Chettri, S. Manivannan, and V. R. Muddarsu, "Nutrient and elemental composition of wild edible ferns of the Himalaya," American fern journal, vol. 108, no. 3, pp. 95-106, 2018.

[27] D. Irawan, C. H. Wijaya, S. H. Limin, Y. Hashidoko, M. Osaki, and I. P. Kulu, "Ethnobotanical study and nutrient potency of local traditional vegetables in Central Kalimantan," Tropics, vol. 15, no. 4, pp. 441-448, 2006.

[28] H. S. Jasim, M. Idris, A. Abdullah, and A. Kadhum, "Determination of heavy metals in soil and different parts of Diplazium esculentum (medicinal fern)," AIP Conference Proceedings, vol. 1614, pp. 713-718, 2014.

[29] Y. Koniyo, C. Lumenta, A. H. Olii, and R. O. Mantiri, “The characteristic and nutrients concentrated leaves of vegetable fern (Diplazium esculentum (Retz.) Swartz) live in dofferent locations," Journal of Physics: Conference Series, vol. 1387, article 012003, 2019.

[30] M. Ogle, H. T. A. Dao, G. Mulokozi, and L. Hambraeus, "Micronutrient composition and nutritional importance of gathered vegetables in Vietnam," International Journal of Food Sciences and Nutrition, vol. 52, no. 6, pp. 485-499, 2001.

[31] R. H. Della, C. H. Wijaya, Y. Hashidoko et al., "Concentration of some trace elements in two wild edible ferns, Diplazium esculentum and Stenochlaena palutris, inhabiting tropical peatlands under different environments in Central Kalimantan," Eurasian Journal of Forest Research, vol. 20, pp. 11-20, 2017.

[32] A. Sayeed, M. S. Islam, M. N. Uddin et al., "Nutritional status of exotic and indigenous vegetables," International Journal of Vegetable Science, vol. 27, no. 1, pp. 86-95, 2020.

[33] J. V. V. Tongco, R. A. P. Villaber, R. M. Aguda, and R. A. Razal, "Nutritional and phytochemical screening, and total phenolic and flavonoid content of Diplazium esculentum (Retz.) Sw. from Philippines," Journal of Chemical and Pharmaceutical Research, vol. 6, pp. 238-242, 2014.

[34] S. N. K. Zihad, Y. Gupt, S. J. Uddin et al., "Nutritional value, micronutrient and antioxidant capacity of some green leafy vegetables commonly used by southern coastal people of Bangladesh," Heliyon, vol. 5, no. 11, article e02768, 2019. 
[35] Y. Koniyo, C. Lumenta, A. Olii, R. Mantiri, and N. Pasisingi, "Nutrition of local wild edible fern (Diplazium esculentum) leaves," IOP Conference Series: Earth and Environmental Science, vol. 637, article 012008, 2021.

[36] O. Neamsuvan and T. Ruangrit, "A survey of herbal weeds that are used to treat gastrointestinal disorders from southern Thailand: Krabi and Songkhla provinces," Journal of Ethnopharmacology, vol. 209, pp. 318-327, 2017.

[37] M. M. Rahman, G. Z. H. Masum, P. Sharkar, and S. N. Sima, "Medicinal plant usage by traditional medical practitioners of rural villages in Chuadanga district, Bangladesh," International Journal of Biodiversity Science, Ecosystem Services \& Management, vol. 9, no. 4, pp. 330-338, 2013.

[38] S. Shil and M. D. Choudhury, "Ethnomedicinal importance of pteridophytes used by Reang tribe of Tripura, North East India," Ethnobotanical Leaflets, vol. 2009, p. 10, 2009.

[39] R. Pegu, J. Gogoi, A. K. Tamuli, and R. Teron, "Ethnobotanical study of wild edible plants in Poba Reserved Forest, Assam, India: multiple functions and implications for conservation," Research Journal of Agriculture and Forestry Sciences, vol. 2320, article 6063, 2013.

[40] G. Kunkel, Plants for Human Consumption, Koeltz Scientific Books, Koenigstein, 1984.

[41] K. Chandra, B. Nautiyal, and M. Nautiyal, "Ethno-botanical resources as supplementary foods and less known wild edible fruits in district Rudraprayag, Uttarakhand, India," Journal of Human Ecology, vol. 42, no. 3, pp. 259-271, 2013.

[42] A. K. Das, B. Dutta, and G. Sharma, "Medicinal plants used by different tribes of Cachar district, Assam," Indian Journal of Traditional Knowledge, vol. 7, pp. 446-454, 2008.

[43] N. A. Farooquee, B. Majila, and C. Kala, "Indigenous knowledge systems and sustainable management of natural resources in a high altitude society in Kumaun Himalaya, India," Journal of Human Ecology, vol. 16, no. 1, pp. 33-42, 2004.

[44] A. Sen and P. Ghosh, "A note on the ethnobotanical studies of some pteridophytes in Assam," Indian Journal of Traditional Knowledge, vol. 10, pp. 292-295, 2011.

[45] S. Yumkham and P. Singh, "Less known ferns and fern-allies of Manipur with ethnobotanic uses," Indian Journal of Traditional Knowledge, vol. 10, pp. 287-291, 2011.

[46] S. Akter, M. M. Hossain, I. Ara, and P. Akhtar, "Investigation of in vitro antioxidant, antimicrobial and cytotoxic activity of Diplazium esculentum (Retz.) Sw," International Journal of Advances in Pharmacy, Biology and Chemistry, vol. 3, pp. 723-733, 2014.

[47] R. Kagyung, P. Gajurel, P. Rethy, and B. Singh, "Ethnomedicinal plants used for gastro-intestinal diseases by Adi tribes of Dehang-Debang Biosphere Reserve in Arunachal Pradesh," Indian Journal of Traditional Knowledge, vol. 9, pp. 496501, 2010.

[48] M. O. Nwosu, "Ethnobotanical studies on some pteridophytes of Southern Nigeria," Economic Botany, vol. 56, no. 3, pp. 255-259, 2002.

[49] Y. Uprety, E. K. Boon, R. C. Poudel et al., "Non-timber forest products in Bardiya district of Nepal: indigenous use, trade and conservation," Journal of Human Ecology, vol. 30, no. 3, pp. 143-158, 2010.

[50] V. B. Amoroso, A. Lagumbay, R. A. Mendez, R. Y. Dela Cruz, and A. P. Villalobos, "Bioactives in three Philippine edible ferns," Asia Life Sciences, vol. 23, pp. 445-454, 2014.
[51] M. D. Astuti, E. M. Kuntorini, and F. E. P. Wisuda, "Isolasi dan identifikasi terpenoid dari fraksi n-butanol herba lampasau (Diplazium esculentum Swartz)," Jurnal Kimia Valensi, vol. 4, 2014.

[52] H. Bhatia, Y. P. Sharma, R. Manhas, and K. Kumar, "Traditionally used wild edible plants of district Udhampur, J\&K, India," Journal of Ethnobiology and Ethnomedicine, vol. 14, no. 1, p. 73, 2018.

[53] A. Thakur, S. Singh, and S. Puri, "Exploration of wild edible plants used as food by Gaddis-a tribal community of the Western Himalaya," The Scientific World Journal, vol. 2020, Article ID 6280153, 6 pages, 2020.

[54] S. Rout, T. Panda, and N. Mishra, "Ethnomedicinal studies on some pteridophytes of similipal biosphere reserve, Orissa, India," International Journal of Medicine and Medical Sciences, vol. 1, pp. 192-197, 2009.

[55] R. Jahan, K. Jannat, J. F. Shoma, M. A. Khan, H. U. Shekhar, and M. Rahmatullah, "Drug discovery and herbal drug development: a special focus on the anti-diarrheal plants of Bangladesh," in Herbal Medicine in India, pp. 363-400, Springer, 2020.

[56] M. Rai, "Ethno-medical studies of patalkot and tamiya (distt. Chhindwara) MP-plants used as tonic," Ancient Science of Life, vol. 7, p. 119, 1987.

[57] S. Pradhan, S. Manivannan, and J. P. Tamang, "Proximate, mineral composition and antioxidant properties of some wild leafy vegetables," Journal of Scientific and Industrial Research, vol. 74, pp. 155-159, 2015.

[58] S. Shrestha, "Use and chemical analysis of wild food in protected areas of Nepal," Nepalese Journal of Agricultural Sciences, vol. 9, pp. 103-106, 2011.

[59] A. K. Rai, R. M. Sharma, and J. P. Tamang, "Food value of common edible wild plants of Sikkim," Journal of Hill Research, vol. 18, pp. 99-103, 2005.

[60] B. Naik, V. K. Maurya, V. Kumar, V. Kumar, S. Upadhyay, and S. Gupta, "Phytochemical analysis of Diplazium esculentum reveals the presence of medically important components," Current Nutrition \& Food Science, vol. 17, no. 2, pp. 210-215, 2021.

[61] R. Ghanbari, F. Anwar, K. M. Alkharfy, A.-H. Gilani, and N. Saari, "Valuable nutrients and functional bioactives in different parts of olive (Olea europaea L.) - a review," International Journal of Molecular Sciences, vol. 13, no. 3, pp. 3291-3340, 2012.

[62] G. Muhammad, M. A. Hussain, I. Jantan, and S. N. A. Bukhari, "Mimosa pudica L., a high-value medicinal plant as a source of bioactives for pharmaceuticals," Comprehensive Reviews in Food Science and Food Safety, vol. 15, no. 2, pp. 303-315, 2016.

[63] S. Chawla, S. Chawla, V. Ram, A. Semwal, and R. Singh, "Analgesic activity of medicinally important leaf of Diplazium esculentum," African Journal of Pharmacy and Pharmacology, vol. 9, pp. 628-632, 2015.

[64] J. Choudhury, S. Majumdar, S. Roy, and U. Chakraborty, "Antioxidant activity and phytochemical screening of two edible wetland pteridophytes Diplazium esculentum (Retz) Sw and Marsilea minuta L.-a comparative study," World Journal of Pharmaceutical and Medical Research, vol. 3, pp. 195-203, 2017.

[65] B. Das, T. Paul, K. G. Apte, R. Chauhan, and R. C. Saxena, "Evaluation of antioxidant potential \& quantification of 
polyphenols of Diplazium esculentum Retz. with emphasis on its HPTLC chromatography," Journal of Pharmacy Research, vol. 6, no. 1, pp. 93-100, 2013.

[66] H. Halimatussakdiah, U. Amna, and P. Wahyuningsih, "Preliminary phytochemical analysis and larvicidal activity of edible fern (Diplazium esculentum (retz.) sw.) extract against culex," Jurnal Natural, vol. 18, no. 3, pp. 141-147, 2018.

[67] J. A. Junejo, A. Ghoshal, P. Mondal et al., "In-vivo toxicity evaluation and phytochemical, physicochemical analysis of Diplazium esculentum (Retz.) Sw. leaves a traditionally used North-Eastern Indian vegetable," Advances in Bioresearch, vol. 6, no. 5, 2015.

[68] S. Khatoniar, M. S. Barooah, and I. C. Baruah, "Qualitative and quantitative phytochemical assessment and antioxidant activity of selected green leafy vegetables of Assam," Journal of Pharmacognosy and Phytochemistry, vol. 7, pp. 17621765, 2018.

[69] J. Tabiano and Y. Deliman, "In vitro inhibitory activity of Atuna racemosa, Euphorbia hirta and Diplazium esculentum juices against $\alpha$-amylase and $\alpha$-glucosidase," International Seminar on Science and Technology, vol. 2014, p. 89, 2014.

[70] M. Caldwell, "Ascorbic acid content of Malaysian leaf vegetables," Ecology of Food and Nutrition, vol. 1, no. 4, pp. 313317, 1972.

[71] S. Srivastava, S. Srivastava, V. Saksena, and S. Nigam, “A flavanone glycoside from Diplazium esculentum," Phytochemistry, vol. 20, no. 4, p. 862, 1981.

[72] I. N. Hayati, A. Aminah, S. Mamot, I. N. Aini, and H. M. N. Lida, "Physical characteristics of modified milkfat in highmelting fat preparation," International Journal of Food Sciences and Nutrition, vol. 53, no. 1, pp. 43-54, 2002.

[73] L. S. Ching and S. Mohamed, "Alpha-tocopherol content in 62 edible tropical plants," Journal of Agricultural and Food Chemistry, vol. 49, no. 6, pp. 3101-3105, 2001.

[74] K. H. Miean and S. Mohamed, "Flavonoid (myricetin, quercetin, kaempferol, luteolin, and apigenin) content of edible tropical plants," Journal of Agricultural and Food Chemistry, vol. 49, no. 6, pp. 3106-3112, 2001.

[75] S. Pathania, P. Kumar, S. Singh et al., "Detection of ptaquiloside and quercetin in certain Indian ferns," Current Science, vol. 102, no. 12, pp. 1683-1691, 2012.

[76] R. Somvanshi, D. Lauren, B. Smith et al., "Estimation of the fern toxin, ptaquiloside, in certain Indian ferns other than bracken," Current Science, vol. 102, no. 12, pp. 1547-1552, 2006.

[77] A. Wali, S. Sharma, M. Walia et al., "Two edible ferns of Western Himalaya: a comparative in vitro nutritional assessment, antioxidant capacity and quantification of lutein by UPLC-DAD," International Journal of Food and Nutritional Sciences, vol. 5, p. 9, 2016.

[78] M. Watanabe, T. Miyashita, and H. P. Devkota, "Phenolic compounds and ecdysteroids of Diplazium esculentum (Retz.) Sw. (Athyriaceae) from Japan and their chemotaxonomic significance," Biochemical Systematics and Ecology, vol. 94, article 104211, 2021.

[79] Y. Kumar, D. N. Yadav, T. Ahmad, and K. Narsaiah, "Recent trends in the use of natural antioxidants for meat and meat products," Comprehensive Reviews in Food Science and Food Safety, vol. 14, no. 6, pp. 796-812, 2015.

[80] S. Maqsood, S. Benjakul, A. Abushelaibi, and A. Alam, "Phenolic compounds and plant phenolic extracts as natural anti- oxidants in prevention of lipid oxidation in seafood: a detailed review," Comprehensive Reviews in Food Science and Food Safety, vol. 13, no. 6, pp. 1125-1140, 2014.

[81] A. Nur, F. Khairul, M. Nuradibah, and S. Noor, "Optimization of Diplazium esculentum extract using pressurized hot water extractor by Box-Behnken design of experiments and its antioxidative behavior," IOP Conference Series: Materials Science and Engineering, vol. 429, article 012064, 2018.

[82] J. G. Junejo, G. Gogoi, J. Islam et al., "Exploration of antioxidant, antidiabetic and hepatoprotective activity of Diplazium esculentum - A wild edible plant from North Eastern India," Future Journal of Pharmaceutical Sciences, vol. 4, no. 1, pp. 93-101, 2018.

[83] S. Roy, S. Dutta, and T. K. Chaudhuri, "In vitro assessment of anticholinesterase and NADH oxidase inhibitory activities of an edible fern, Diplazium esculentum," Journal of Basic and Clinical Physiology and Pharmacology, vol. 26, no. 4, pp. 395-401, 2015.

[84] V. Rana, R. Bachheti, S. Gogoi, P. Gupta, and G. Joshi, "Physicochemical, functional and antioxidant properties of Diplazium esculentum leaf protein concentrate," Current Traditional Medicine, vol. 1, no. 2, pp. 145-158, 2015.

[85] E. P. Jayag and A. D. Acabal, "In vitro free radical scavenging activity of Bago (Gnetum gnemon Linn.), Pako (Diplazium esculentum (Retz.) Sw.) and Saluyot (Corchorus olitorius Linn.) leaf extracts," Journal of Society and Technology, vol. 4, pp. 17-24, 2014.

[86] S. Gupta, M. Ghosal, R. Biswas, B. Saha, A. Das, and P. Mandal, "Evaluation of in vitro antioxidant activity of methanolic extracts of some ferns from Mawsynram of Meghalaya, India," International Journal of Current Science, vol. 12, pp. E87-E97, 2014.

[87] S. Roy, B. Hazra, N. Mandal, and T. K. Chaudhuri, “Assessment of the antioxidant and free radical scavenging activities of methanolic extract of Diplazium esculentum," International Journal of Food Properties, vol. 16, no. 6, pp. 13511370, 2013.

[88] A. Kaushik, C. Jijta, J. J. Kaushik, R. Zeray, A. Ambesajir, and L. Beyene, "FRAP (Ferric reducing ability of plasma) assay and effect of Diplazium esculentum (Retz) Sw.(a green vegetable of North India) on central nervous system," Indian Journal of Natural Products and Resources, vol. 3, pp. 228-231, 2012.

[89] T. Seal, "Antioxidant activity of some wild edible plants of Meghalaya state of India: a comparison using two solvent extraction systems," International Journal of Nutrition and Metabolism, vol. 4, pp. 51-56, 2012.

[90] S. Nanasombat and N. Teckchuen, "Antimicrobial, antioxidant and anticancer activities of Thai local vegetables," Journal of Medicinal Plants Research, vol. 3, pp. 443-449, 2009.

[91] S. Wong, L. Leong, and J. Williamkoh, "Antioxidant activities of aqueous extracts of selected plants," Food Chemistry, vol. 99, no. 4, pp. 775-783, 2006.

[92] Z. Sheriff, "Modern Herbal Therapy for Common Ailments," in Nature Pharmacy Series Vol. 1. Spectrum Book Limited, Ibadan, pp. 9-84, Nigeria in Association with Safari Books (Export) Limited, UK, 2001.

[93] J. L. Rios and M. C. Recio, "Medicinal plants and antimicrobial activity," Journal of Ethnopharmacology, vol. 100, no. 1-2, pp. 80-84, 2005.

[94] Z. Zakaria, S. Sanduran, and S. Sreenivasan, "Antifungal activity of the edible ferns: application for public health," 
International Journal of the Humanities, vol. 8, no. 8, pp. 113-118, 2010.

[95] M. Mackeen, A. Ali, S. El-Sharkawy et al., "Antimicrobial and cytotoxic properties of some Malaysian traditional vegetables (ulam)," International Journal of Pharmacognosy, vol. 35, no. 3, pp. 174-178, 1997.

[96] S. Amit, K. Sunil, S. Bhatt, and N. Arvind, "Antibacterial activity of Diplazium esculentum (Retz.) Sw," Pharmacognosy Journal, vol. 3, no. 21, pp. 77-79, 2011.

[97] G. P. Shing, C. L. Wen, T. S. Wei, O. H. Chooi, K. K. Soo, and S. N. Weng, "Antifungal and antibacterial properties of three medicinal plants from Malaysia," Pharmacognosy Communications, vol. 3, no. 2, pp. 75-81, 2013.

[98] M. O. Ullah, M. Haque, K. F. Urmi et al., "Anti-bacterial activity and brine shrimp lethality bioassay of methanolic extracts of fourteen different edible vegetables from Bangladesh," Asian Pacific Journal of Tropical Biomedicine, vol. 3, no. 1, pp. 1-7, 2013.

[99] S. S. Irudayaraj, C. Sunil, V. Duraipandiyan, and S. Ignacimuthu, "Antidiabetic and antioxidant activities of Toddalia asiatica (L.) Lam. leaves in streptozotocin induced diabetic rats," Journal of Ethnopharmacology, vol. 143, no. 2, pp. 515-523, 2012.

[100] S. Roy, S. Tamang, P. Dey, and T. K. Chaudhuri, “Assessment of the immunosuppressive and hemolytic activities of an edible fern, Diplazium esculentum," Immunopharmacology and Immunotoxicology, vol. 35, no. 3, pp. 365-372, 2013.

[101] S. Roy and T. K. Chaudhuri, "Assessment of Th1 and Th2 cytokine modulatory activity of an edible fern, Diplazium esculentum," Food and Agricultural Immunology, vol. 26, no. 5, pp. 690-702, 2015.

[102] Y. X. You, S. Shahar, H. Haron, H. M. Yahya, and N. C. Din, "Relationship between traditional Malaysian vegetables (Ulam) intake and cognitive status among middle-aged adults from low cost residential areas," Jurnal Sains Kesihatan Malaysia (Malaysian Journal of Health Sciences), vol. 17, 2020.

[103] A. Rahmat, V. Kumar, L. M. Fong, S. Endrini, and H. A. Sani, "Determination of total antioxidant activity in three types of local vegetables shoots and the cytotoxic effect of their ethanolic extracts against different cancer cell lines," Asia Pacific Journal of Clinical Nutrition, vol. 12, no. 3, pp. 292-295, 2003.

[104] G. N. Kumar, "Studies on pathological effects of linguda (Diplazium esculentum, Retz.) in laboratory rats and guinea pigs," Indian Journal of Veterinary Pathology, vol. 28, p. 149, 2004.

[105] S. Roy and T. K. Chaudhuri, "Toxicological assessment of Diplazium esculentum on the reproductive functions of male Swiss albino mouse," Drug and Chemical Toxicology, vol. 40, no. 2, pp. 171-182, 2017.

[106] B. Das, T. Paul, K. Apte, P. Parab, R. Chauhan, and R. Saxena, "Antianaphylactic and mast cell stabilizing activity of Diplazium esculentum Retz. on sensitized wistar rats," Inventi Impact Ethnopharmacol, vol. 3, pp. 136-141, 2012.

[107] M. Zaini, A. Biworo, and K. Anwar, "Uji efek antiinflamasi ekstrak etanol herba lampasau (Diplazium esculentum Swartz) terhadap mencit jantan yang diinduksi karagenin$\Lambda$," Jurnal Pharmascience, vol. 3, 2017.

[108] N. A. Zainol, H. A. Aziz, and N. A. Lutpi, "Diplazium esculentum leaf extract as coagulant aid in leachate treatment," In
Proceedings of AIP Conference Proceedings, vol. 1835, no. 1, article 020034, 2017.

[109] A. S. Semwal, "MF In-vitro anthelmintic activity of Diplazium esculentum (Retz.) Swiss rhizome extract," Journal of Pharmacognosy and Phytochemistry, vol. 1, pp. 84-87, 2012.

[110] B. Paul, B. Bhuyan, D. D. Purkayastha, and S. S. Dhar, "Green synthesis of silver nanoparticles using dried biomass of Diplazium esculentum (retz.) sw. and studies of their photocatalytic and anticoagulative activities," Journal of Molecular Liquids, vol. 212, pp. 813-817, 2015.

[111] I. Norhayati, K. Getha, J. M. Haffiz et al., "In vitro antitrypanosomal activity of Malaysian plants," Journal of Tropical Forest Science, vol. 25, no. 1, pp. 52-59, 2013. 